Pathologe 2021 · 42:183-187 https://doi.org/10.1007/s00292-020-00899-1 Angenommen: 16. Dezember 2020 Online publiziert: 1. Februar 2021 (c) Springer Medizin Verlag $\mathrm{GmbH}$, ein Teil von Springer Nature 2021

\section{Schwerpunktherausgeber}

W. Roth, Mainz

P. Boor, Aachen

K. Amann' $\cdot$ P. Boor ${ }^{2}$ T. Wiech ${ }^{3}$ J. Singh ${ }^{4}$ E. Vonbrunn' $\cdot$ A. Knöll ${ }^{5} \cdot$ M. Hermann ${ }^{4}$. M. Büttner-Herold ${ }^{1}$. C. Daniel ${ }^{1}$. A. Hartmann ${ }^{6}$

${ }^{1}$ Abt. Nephropathologie, Universitätsklinikum Erlangen, Erlangen, Deutschland

${ }^{2}$ Institut für Pathologie \& Medizinische Klinik II (Nephrologie), Sektion Translationale Nephropathologie, Universitätsklinikum der RWTH Aachen, Aachen, Deutschland

${ }^{3}$ Institut für Pathologie, Sektion Nephropathologie, Universitätsklinikum Hamburg-Eppendorf, Hamburg, Deutschland

${ }^{4}$ Medizinische Klinik 3, Universitätsklinikum Erlangen, Erlangen, Deutschland

${ }^{5}$ Virologie, Universitätsklinikum Erlangen, Erlangen, Deutschland

${ }^{6}$ Pathologisches Institut, Universitätsklinikum Erlangen, Erlangen, Deutschland

\title{
COVID-19-Auswirkungen auf die Niere
}

4. systemische Endothelschädigung, Thrombosen und Multiorganschädigung [4].

Seit Beginn der COVID-19-Pandemie hat die Kenntnis über den Verlauf, die Organbeteiligung und die Pathophysiologie der Infektion mit dem „severe acute respiratory syndrome coronavirus $2^{\text {" }}$ (SARS-CoV-2) rasant zugenommen. Bei einer SARS-CoV-2-Infektion binden die Spikeproteine des Virus an ,angiotensin converting enzyme 2" (ACE2), einen Rezeptor auf der Zelloberfläche, und werden durch membranständige Proteasen gespalten, sodass das Virus mit der Wirtszelle verschmelzen kann [1, 2]. SARS-CoV-2 repliziert zunächst in den Atemwegsepithelien, wird dann in die Blutbahn ausgeschwemmt und im Rahmen dieser Virämie kommt es zur Infektion von Endothelien [3]. Weitere Ausführungen zu SARS-CoV-2 und der Lungenbeteiligung finden sich in anderen Beiträgen dieses Sonderheftes.

Die COVID-19-Erkrankung kann bei schwerem Verlauf in 4 Stadien ablaufen:

1. virale Infektion und Replikation,

2. Virusdisseminierung,

3. schwere systemische Entzündung und

Die englische Version dieses Beitrages ist unter https://doi.org/10.1007/s00292-020-00900-x zufinden.
Neben dem Respirationstrakt und dem Gerinnungssystem $[5,6]$ können in diesem Rahmen auch andere Organe und hier insbesondere die Niere in Mitleidenschaft gezogen werden [7]. Klinisch manifestiert sich dies v. a. in Form eines akuten Nierenversagens (ANV), dessen Pathogenese mutmaßlich multifaktoriell ist. Neben der direkten Infektion von Nierenzellen über den ACE2-Rezeptor [8], der auf proximalen Tubulusepithelien, parietalen Epithelzellen und teils auch Podozyten exprimiert wird und möglicherweise mittels ACE2-Hemmern inhibiert werden kann [9], kommen sicher auch sekundäre Veränderungen, z. B. bei Gerinnungsstörungen und Ischämie, in Betracht. Einige dieser Aspekte sollen im Folgenden näher beleuchtet werden.

\section{Nierensymptomatik bei COVID- 19-erkrankten hospitalisierten Patienten}

Nierensymptome, insbesondere das ANV als häufigstes klinisches Symptom der Nierenbeteiligung, werden in der Literatur in variablem Ausmaß berichtet [10]. So fanden Richardson et al. [11] ein ANV bei mehr als $20 \%$ der in einem New Yorker Krankenhaus behandelten Patienten $(n=5700)$. Von diesen benötigten 3,2 \% eine Nierenersatztherapie. In einer Studie aus Wuhan [12] wird berichtet, dass ca. $20 \%$ der COVID-19Intensivpatienten mit ANV auch dialysepflichtig wurden. In einer weitere chinesischen Arbeit [13] an 701 Patienten wurde bei $43,9 \%$ der hospitalisierte COVID-19-Patienten eine Proteinurie, bei $26,7 \%$ eine Hämaturie und bei $13,1 \%$ eine glomeruläre Filtrationsrate (GFR) von $<60 \mathrm{ml} / \mathrm{min} / 1,73 \mathrm{~m}^{2}$ beschrieben. Im Folgenden entwickelten 5\% dieser Patienten dann ein ANV. COVID-19Patienten, die ein ANV entwickeln, haben ein deutlich schlechteres Outcome mit höherem Risiko zu versterben.

\section{COVID-19-Nierensymptomatik bei chronisch nierenkranken und nierentransplantierten Patienten}

Ähnlich wie bei einem ANV anderer Genese scheint eine vorbestehende chronische Nierenerkrankung (,chronic kidney disease", CKD) mit einem erhöhten Risiko eines schwereren Verlaufs der COVID-19-Erkrankung bzw. einer erhöhten Mortalität einherzugehen [13, 14]. Aus dem österreichischen Dialyseund Nierentransplantationsregister ist bekannt, dass zwischen Mitte März und Anfang April 2020 die Zahl COVID-19positiver Patienten in diesem Register drastisch in die Höhe ging, um dann auf einem hohen Plateau zu verharren und 


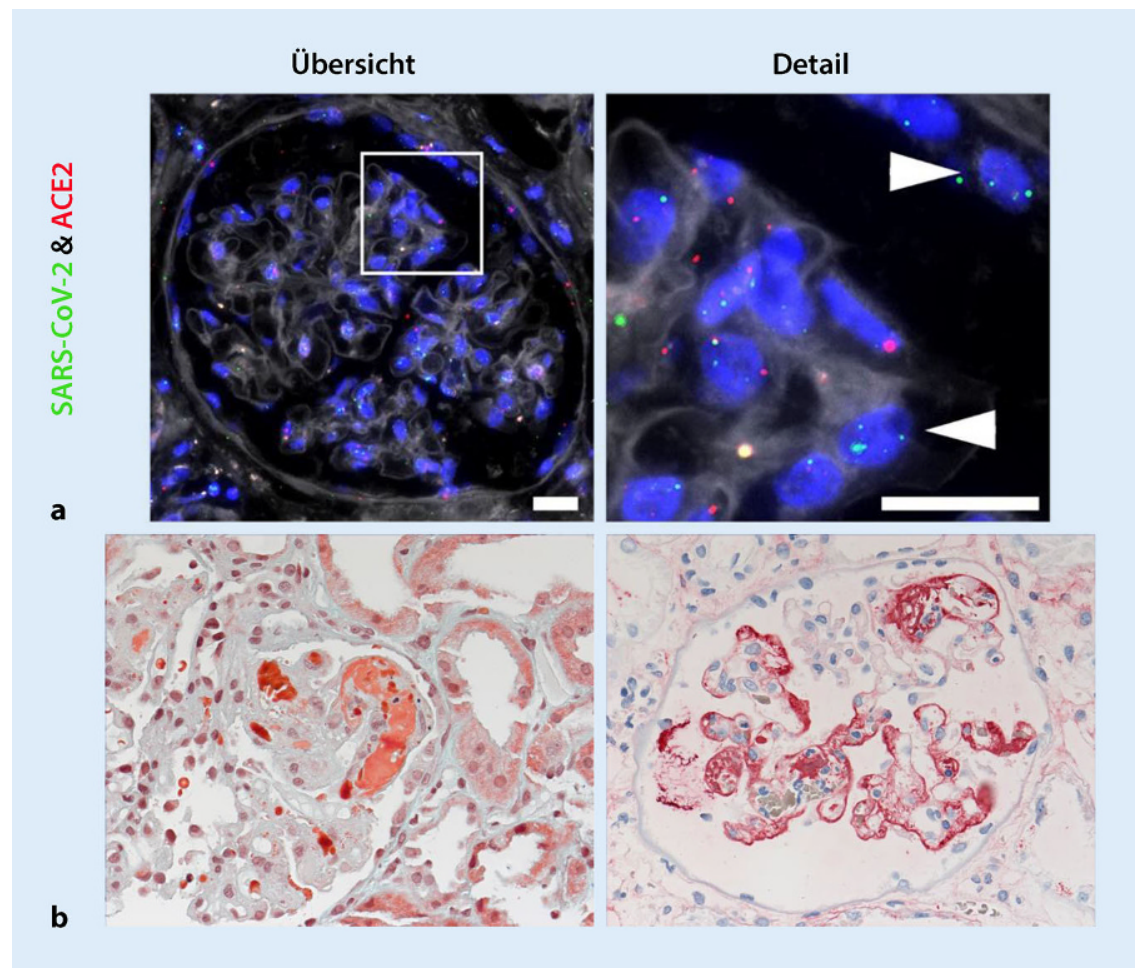

Abb. 1 \SARS-CoV-2-Virusnachweis in der Niere und charakteristische glomeruläre Veränderungen. a SARS-CoV-2-Virusnachweis in der Niere. Fluoreszenz-in-situ-Hybridisierung (FISH) zeigt die RNAExpression von SARS-CoV-2-Virus (grün; Pfeile) und seines Rezeptors "angiotensin-converting enzyme 2" (ACE2; rot) im Glomerulus der Niere eines COVID-19-Patienten. Maßstabsbalken $=20 \mu \mathrm{m}$. (Aus Amann et al. [36]). b Nierenhistologie eines 38 Jahre alten Patienten mit COVID-19-Erkrankung und akutem Nierenversagen. Links: In der Masson-Goldner-Färbung stellen sich frische, wandhaftende Fibrinthromben (orange) in den glomerulären Kapillaren dar. Rechts: In der Immunhistologie für Fibrinogen/Fibrin finden sich wandhaftende Präzipitate (rot) in zahlreichen glomerulären Kapillarlichtungen

jüngst wieder etwas anzusteigen. Die SARS-CoV-2-infizierten nierenkranken Patienten zeigten insgesamt eine hohe Sterblichkeit: $27,9 \%$ der infizierten Dialysepatienten und $6 \%$ der nierentransplantierten Patienten verstarben laut diesem Register an bzw. mit einer COVID-19-Erkrankung. Von den insgesamt 18 Todesfälle wurden $14(77,7 \%)$ als direkte Folge der SARS-CoV-2-Infektion gewertet.

Diese Befunde werden auch durch die Daten des EDTA-Registers bestätigt [14, S. 1540-1548], wo insbesondere auch die mit $44,3 \%$ sehr hohe Sterblichkeit an COVID-19 in der Gruppe der nierentransplantierten Patienten $\geq 75$ Jahre betont wird. Dies bedeutet eine 1,28fach erhöhte Sterblichkeit im Vergleich zu einer gematchten Dialysekohorte, sodass nierentransplantierte Patienten damit eine besonders vulnerable Population darstellen, was mutmaßlich auf die zugrunde liegende Nierenerkrankung und die vielfältigen Komorbiditäten zurückzuführen ist. Neben einer erhöhten Sterblichkeit scheint die COVID-19Erkrankung bei nierentransplantierten Patienten aber auch andere funktionelle Auswirkungen zu haben, wie zahlreiche mittlerweile publizierte Fallberichte darstellen.

\section{Morphologische Nierenverän- derungen und Pathophysiologie der Nierenschädigung bei COVID-19}

Spezifische Veränderungen bzw. charakteristische morphologische Läsionen wie bei anderen Viruserkrankungen, z.B. ein diffuses interstitielles Ödem und interstitielle Erythrozytenextravasate im Rinden-Mark-Übergangsbereich bei Hantavirus-Nephropathie, oder charakteristische nukleäre Viruseinschlusskörperchen wie bei Polyoma- und Zytomegalovirus-Infektion, liegen bei SARS-
CoV-2-Infektion nicht vor bzw. sind bislang nicht beschrieben [17]. In den bisher veröffentlichten Studien sowie unseren eigenen Beobachtungen ist an der Niere ein akuter Tubulusepithelschaden unterschiedlichen Ausmaßes mit dilatierten Tubuli, abgeflachten oder vakuolisierten Epithelzellen und eingedicktem Material im Tubuluslumen das dominierende histologische Merkmal. Su et al. [18] untersuchten Nierengewebe von 26 COVID-19-Patienten, von denen 9 klinische Hinweise auf eine Nierenschädigung hatten. Ein akuter Schaden der proximalen Tubuli mit vermehrter Vakuolisierung bis hin zur Epithelnekrose und Erythrozytenkonglomerate in den glomerulären und peritubulären Kapillaren waren die lichtmikroskopischen Hauptbefunde. Wenige Fälle wiesen auch pigmentierte Zylinder auf. In der Autopsiestudie von Menter et al. [19] wurden die Nieren von 18 verstorbenen COVID19-Patienten analysiert und ebenfalls ein akuter Tubulusschaden mit aufgeweiteten Lichtungen, abgeflachtem Epithel und interstitiellem Ödem als häufigster Befund beschrieben. Zusätzlich wiesen 3 Patienten kleine Fibrinthromben in den glomerulären Kapillaren im Sinne einer disseminierten intravasalen Gerinnung („disseminated intravascular coagulation“, DIC) auf, wie sie im Kontext der Lungenbeteiligung bei COVID-19 ebenfalls beschrieben wurde. Einblutungen, entzündliche Gefäßveränderungen oder interstitielle Nephritiden wurden in beiden Studien nicht beobachtet, was jedoch eine pathophysiologische Beteiligung inflammatorischer Prozesse wie verstärkter Zytokin- oder Komplementaktivierung nicht ausschließt. Diese Faktoren sind allerdings im Gewebe schwierig spezifisch darzustellen.

Ganz aktuell sind mehrere Arbeiten in internationalen nephrologischen Journalen erschienen, die sich mit Autopsieund Nierenbiopsiebefunden bei SARS$\mathrm{CoV}$-2-positiven Patienten beschäftigen [20-22]. Hier wurden wiederum v. a. akute Tubulusepithelschäden gefunden und ganz überwiegend keine charakteristischen Viruspartikel nachgewiesen, sodass in diesen Untersuchungen vieles für eine indirekte Ursache des ANV spricht. In einer jüngst publizierten Arbeit un- 
tersuchten Werion und Kollegen [23] 49 hospitalisierte Patienten und fanden bei einer Untergruppe eine spezifische Dysfunktion der proximalen Tubuli, die unabhängig von etwaigen Vorerkrankungen war. Strukturell zeigten sich Tubulusschäden mit Verlust des Bürstensaums, akuten Tubulusnekrosen, intratubulärem Debris und verminderter Expression des Endozytoserezeptors Megalin im Bürstensaum. Elektronenmikroskopisch wurden virusähnliche Partikel in Vakuolen oder Zisternen des endoplasmatischen Retikulums (ER) gefunden. Überlegungen zu Pathophysiologie und Behandlung des ANV bei COVID-19 wurden u. a. von Ronco und Reis [24] publiziert. Hier spielen neben dem direkten Virusbefall ortsständiger Nierenzellen über den ACE2Rezeptor mehrere indirekte Faktoren eine Rolle, die mit einer sekundären Nierenschädigung einhergehen. So kann es im Rahmen der sehr häufigen Lungenbeteiligung bei COVID-19 zu einer verminderten Sauerstoffsättigung des Blutes und dadurch zu einer ischämischen Schädigung v. a. der sehr energieabhängigen proximalen Tubulusabschnitte kommen. Weiterhin ist von einer virusbedingten Aktivierung des Komplementsystems auszugehen, die systemisch oder lokal an der Niere über die Freisetzung von Mediatoren wie Zytokinen zur Inflammation und Zellschädigung am Tubulusapparat führt [25]. Eine derartige Komplementaktivierung könnte gleichzeitig auch die Schädigung anderer Organe verstärken und die Hyperkoagulopathie begünstigen, durch die dann arterielle Fibrinthromben und DIC oder eine Endothelschädigung mit konsekutiver thrombotischer Mikroangiopathie (• Abb. 1b) entstehen und zu einer Minderperfusion der Nieren führen können. Arterielle Thromben in pulmonalen Gefäßen wiederum führen zu einem erhöhten pulmonalen Widerstand und damit zu einer Rechtsherzbelastung, die ihrerseits bei hypoxygeniertem Blut ein ANV begünstigt. Weiterhin wurde eine pathophysiologische Rolle der sog. neutrophilen extrazellulären Traps (NET) für die Mikrozirkulationsstörung bei schwereren COVID19-Verläufen auch in der Niere beschrieben [26]. COVID-19-Patienten zeigen eine Aktivierung und Degranulation der

Pathologe 2021 - 42:183-187 https://doi.org/10.1007/s00292-020-00899-1

(c) Springer Medizin Verlag GmbH, ein Teil von Springer Nature 2021

\section{K. Amann · P. Boor · T. Wiech · J. Singh · E. Vonbrunn · A. Knöll · M. Hermann · M. Büttner- Herold · C. Daniel · A. Hartmann \\ COVID-19-Auswirkungen auf die Niere}

\section{Zusammenfassung}

Bei einer schweren Coronavirus-Erkrankung2019 (COVID-19) ist neben der Lungenerkrankung selbst das akute Nierenversagen (ANV) eine der häufigsten und schwerwiegendsten Komplikationen. Das SARS-CoV-2-Virus konnte hierbei auch in der Niere nachgewiesen werden. Patienten mit chronischen Nierenerkrankungen (CKD), dialysepflichtige sowie v. a. nierentransplantierte Patienten scheinen eine besonders vulnerable Population darzustellen. Die zunehmende Anzahl SARS-CoV-2infizierter Patienten hat das Interesse an der genauen Pathophysiologie und Morphologie der Nierenschädigung sowie am direkten Virusnachweis in der Niere geweckt, der im Gegensatz zur Lunge insgesamt schwieriger zu führen ist. Hierzu liegen mittlerweile Daten aus Autopsie- und Nierenbiopsiestudien mit unterschiedlichen Patientenzahlen und von sehr unterschiedlicher Qualität vor. Während der Nachweis von SARS-CoV-2-RNA im Nierengewebe mit gut reproduzierbaren Ergebnissen erfolgt, ist insbesondere der Virusnachweis mittels Elektronenmikroskopie schwierig und wird aufgrund zahlreicher Artefakte derzeit kritisch diskutiert. Die genauen direkten oder indirekten Effekte von SARS-CoV-2 auf die Niere sind noch nicht im Detail bekannt und derzeit der Fokus intensiver Forschung.

\section{Schlüsselwörter}

Akuter Nierenschaden - CoronavirusInfektionen - Elektronenmikroskopie . Extrazelluläre Netze · SARS-CoV-2

\section{COVID-19 effects on the kidney}

\section{Abstract}

Apart from pulmonary disease, acute kidney injury (AKI) is one of the most frequent and most severe organ complications in severe coronavirus disease 2019 (COVID-19). The SARS-CoV-2 virus has been detected in renal tissue. Patients with chronic kidney disease (CKD) before and on dialysis and specifically renal transplant patients represent a particularly vulnerable population. The increasing number of COVID-19 infected patients with renal involvement led to an evolving interest in the analysis of its pathophysiology, morphology and modes of virus detection in the kidney. Meanwhile, there are ample data from several autopsy and kidney biopsy studies that differ in the quantity of cases as well as in their quality. While the detection of SARS-CoV-2 RNA in the kidney leads to reproducible results, the use of electron microscopy for visualisation of the virus is difficult and currently critically discussed due to various artefacts. The exact contribution of indirect or direct effects on the kidney in COVID-19 are not yet known and are currently the focus of intensive research.

\section{Keywords}

Acute kidney injury - Coronavirus infections . Electron microscopy - Extracellular traps . SARS-CoV-2 neutrophilen Granulozyten im Blut und eine vermehrte Bildung der NETs, welche wiederum Blutgefäße ganz oder teilweise verschließen und zu Gewebeschäden führen können. Derartige Veränderungen wurden jüngst auch in Nieren verstorbener COVID-19-Patienten nachgewiesen [26]. Auch in einer anderen kürzlich erschienenen Arbeit von SchulteSchrepping et al. [27] von der Deutschen COVID-19 OMICS Initiative (DeCOI) wird auf eine wichtige pathophysiologische Rolle dysfunktioneller neutrophi- ler Granulozyten bzw. einer dysregulierten Immunantwort bei COVID-19-Erkrankung hingewiesen, die auch erklären könnte, warum Patienten mit Komorbiditäten wie Diabetes mellitus und eingeschränkter Immunkompetenz ein erhöhtes Mortalitätsrisiko haben.

Weiterhin wird auf einen möglichen Zusammenhang zwischen proteinurischen glomerulären Erkrankungen und einer SARS-CoV-2-Infektion sowie eine mögliche Assoziation mit dem renalen Hochrisiko-APOL1-Genotyp hingewie- 
sen [28]. Diese und andere Befunde sind auch in einem Übersichtsartikel zusammengefasst [29, 30].

\section{SARS-CoV-2-Erregerdiagnostik am fixierten Nierengewebe}

Das SARS-CoV-2 kann auch im Nierengewebe am sensitivsten und spezifischsten anhand der Virus-RNA nachgewiesen werden. Hierzu wird einerseits die PCR-basierte Methode genutzt, in der man nach Isolation von RNA aus dem Gewebe (frisch oder formalinfixiert und paraffineingebettet) ähnlich wie bei einem Abstrich in einer PCR-Reaktion spezifische RNA-Sequenzen des SARSCoV-2 nachweist. Diese Methode ist insbesondere für den hohen Durchsatz und die Bestätigung einer Infektion gut geeignet. Andererseits kann die VirusRNA direkt am Schnitt durch die Insitu-Hybridisierung (ISH) nachgewiesen werden. Hierzu verwendet man komplementäre Sonden, die sich am histologischen Schnitt spezifisch an die Virus-RNA binden. Diese Bindung kann man mittels Fluoreszenz(FISH)- oder Chromogen(CISH)-Farbstoffen visualisieren [15, 16]. Diese Methodik ist etwas aufwendiger, bietet jedoch eine örtliche und zellspezifische Analyse der Viruslokalisation. Auch ist es dadurch möglich, mittels Doppel- bzw. Mehrfachfärbungen weitere Gene (oder Proteine) parallel in derselben Zelle zu analysieren, wie z.B. den Virusrezeptor ACE2 (- Abb. 1a). Mit diese Methoden gelang es schon früh während der Pandemie zu zeigen, dass SARS-CoV-2-RNA nicht nur in der Lunge, sondern in geringeren Ausmaß auch in verschiedenen anderen Organen, inklusive der Niere, zu finden ist. Eine kürzlich erschienene Arbeit vergleicht die Methoden des Virusnachweis an Paraffinmaterial und konnte ebenfalls zeigen, dass der In-situ-Virusnachweis sowohl auf RNA - als auch auf Proteinebene in der Niere in der Regel schwieriger ist als in der Lungen bzw. seltener ein positives Ergebnis zeigt [31]. In der Autopsiestudie von Braun et al. [32] gelang der SARS-CoV-2-RNA-Nachweis in der Niere in $60 \%$ (38 von 63) der Patienten. Dieser Befund war mit einem höheren Lebensalter, einer höheren Anzahl an
Komorbiditäten und einer verkürzten Überlebenszeit assoziiert.

Weiterhin ist im Rahmen der COVID19-Diagnostik auch die ultrastrukturelle Untersuchung mittels Elektronenmikroskopie (EM) zum Virusnachweis zu erwähnen. Die EM kann in der Nephropathologie (und Kardiopathologie) für den Nachweis bzw. die Bestätigung einer Virusinfektion, wie z. B. CMV oder Polyomavirus, benutzt werden. Obwohl der ultrastrukturelle Nachweis von SARSCoV-2 in humanem Gewebe und insbesondere Autopsiematerial alles andere als trivial ist, haben die EM und auch spezifischere Methoden, wie z.B. die Immunogold-EM oder die korrelative Lichtmikroskopische EM (CLEM), hier einen möglichen diagnostischen Wert [33]. Exzellent bebilderte Arbeiten von Roufosse [34] und Hopfer et al. [35] nehmen kritisch Stellung zu den Problemen des Virusnachweis mittels EM und weisen insbesondere auf verschiedene Artefakte hin, die Viruspartikel imitieren können. Somit ist v. a. bei der Verwendung der EM für den Virusnachweis bzw. der Interpretation elektronenmikroskopischer Bilder und Befunde Vorsicht geboten, da es hier zahlreiche Fallstricke bzw. Strukturen gibt, die Viruspartikeln sehr ähnlich sehen. Eine weiterführende Diskussion der Virus-Nachweismethoden ist in einem weiteren Beitrag in dieser Sonderausgabe zu finden.

Abschließend soll nicht unerwähnt bleiben, dass zusätzlich $\mathrm{zu}$ den eben erwähnten möglicherweise artefaktanfälligen Methoden und technischen Aspekten derzeit insgesamt noch abgewartet werden sollte, welche der in kürzester Zeit erhobenen und teils sehr schnell sowie zumindest in einigen Fällen offenbar auch ohne gründlichen Reviewprozess publizierten Befunde zu COVID-19 und Niere einer kritischen Prüfung an größeren Fallzahlen bzw. mit den entsprechenden Kontrollen standhalten.

\section{Fazit für die Praxis}

\footnotetext{
- Im Verlauf einer schweren Infektion mit SARS-CoV-2 kommt es sowohl bei nierengesunden als v. a. auch bei nierenkranken und nierentransplantier-
}

ten Patienten mit vorbestehendem chronischem Schaden nicht selten zu Nierenfunktionsstörungen und einer akuten Niereninsuffizienz.

- Das akute Nierenversagen ist histomorphologisch gekennzeichnet durch eine schwere diffuse akute Tubulusschädigung.

- Wie es zu der akuten Komponente des Nierenversagens kommt, ist im Einzelnen noch nicht vollständig geklärt bzw. mutmaßlich ist von einer multifaktoriellen Genese auszugehen. Sowohl direkte Viruseffekte über den auf zahlreichen Nierenzellen vorhandenen ACE2-Rezeptor als auch indirekte Effekte, wie z. B. eine gestörte Perfusion oder Zytokin- und Komplementaktivierung, könnten eine Rolle spielen.

- Im Gegensatz zu der sonstigen Nierenbeteiligung bei systemischer Virusinfektion liegt bei SARS-CoV-2 keine akute T-Zell-vermittelte interstitielle Nephritis vor und es sind bislang auch keine spezifischen Glomerulonephritiden berichtet worden.

\section{Korrespondenzadresse}

Prof. Dr. K. Amann

Abt. Nephropathologie, Universitätsklinikum Erlangen

Krankenhausstr. 8-10, 91054 Erlangen,

Deutschland

Kerstin.Amann@uk-erlangen.de

Förderung. Diese Arbeit wurde durch das Bundesministerium für Gesundheit (DeRegCOVID, ZMVI12520COR201), das Bundesministerium für Bildung und Forschung in Rahmen des Netzwerks Universitätsmedizin (DEFEAT PANDEMIcs, 01 KX2021) und die DFG (Projekt Nr. 387509280, SFB1350, TP C02) unterstützt.

\section{Einhaltung ethischer Richtlinien}

Interessenkonflikt. K. Amann, P. Boor, T. Wiech, J.Singh, E. Vonbrunn, A. Knöll, M. Hermann, M. BüttnerHerold, C. Daniel und A. Hartmann geben an, dass kein Interessenkonflikt besteht.

Für diesen Beitrag wurden von den Autoren keine Studien an Menschen oder Tieren durchgeführt. Für die aufgeführten Studien gelten die jeweils dort angegebenen ethischen Richtlinien. 


\section{Literatur}

1. Zhou P et al (2020) A pneumonia outbreak associated with a new coronavirus of probable bat origin. Nature 579(7798):270-273

2. Gheblawi $\mathrm{M}$ et al (2020) Angiotensin-converting enzyme 2: SARS-coV-2 receptor and regulator of the renin-angiotensin system: celebrating the 20 th anniversary of the discovery of ACE2. Circ Res 126(10):1456-1474

3. Gupta A et al (2020) Extrapulmonary manifestations of COVID-19. Nat Med 26(7):1017-1032

4. Cordon-Cardo C et al (2020) COVID-19: staging of a new disease. Cancer Cell 38(5):594-597

5. Wichmann D et al (2020) Autopsy findings and venous thromboembolism in patients with COVID19: a prospective cohort study. Ann Intern Med 173(4):268-277

6. Zou L et al (2020) SARS-CoV-2 viral load in upper respiratory specimens of infected patients. N EnglJ Med 382(12):1177-1179

7. Puelles VG et al (2020) Multiorgan and renal tropism of SARS-coV-2. N Engl J Med 383(6):590-592. https://doi.org/10.1056/NEJMc201140

8. Gavriatopoulou M et al (2020) Organ-specific manifestations of COVID-19 infection. Clin Exp Med. https://doi.org/10.1007/s10238-020-00648-x

9. Allison SJ (2020) SARS-CoV-2 infection of kidney organoids prevented with soluble human ACE2. Nat Rev Nephrol 16(6):316. https://doi.org/10. 1038/s41581-020-0291-8

10. Rubin $S$ et al (2020) Characterization of acute kidney injury in critically ill patients with severe coronavirus disease 2019. Clin Kidney J 13(3):354-361

11. Richardson Setal (2020) Presenting characteristics, comorbidities, and outcomesamong 5700 patients hospitalized with COVID-19 in the New York City area. JAMA 323(20):2052-2059

12. Zhou F et al (2020) Clinical course and risk factors for mortality of adult inpatients with COVID-19 in Wuhan, China: a retrospective cohort study. Lancet 395(10229):1054-1062

13. Cheng Y et al (2020) Kidney disease is associated with in-hospital death of patients with COVID-19. Kidney Int 97(5):829-838

14. Jager KJ et al (2020) Results from the ERAEDTA registry indicate a high mortality due to COVID-19 in dialysis patients and kidney transplant recipients across europe. Kidney Int 98(6):1540-1548. https://doi.org/10.1016/j.kint. 2020.09.006

15. Westhoff TH et al (2020) Allograft infiltration and meningoencephalitis by SARS-CoV-2 in a pancreas-kidney transplant recipient. Am J Transplant 20(11):3216-3220

16. Doevelaar AAN et al (2020) Lessons for the clinical nephrologist: recurrence of nephrotic syndrome induced by SARS-CoV-2. J Nephrol 33(6):1369-1372

17. Remmelink Met al (2020) Unspecific post-mortem findings despite multiorgan viral spread in COVID19 patients. Crit Care 24(1):495

18. Su H et al (2020) Renal histopathological analysis of 26 postmortem findings of patients with COVID19 in China. Kidney Int 98(1):219-227. https://doi. org/10.1016/j.kint.2020.04.003

19. Menter T et al (2020) Postmortem examination of COVID-19 patients reveals diffuse alveolar damage with severe capillary congestion and variegated findings in lungs and other organs suggesting vascular dysfunction. Histopathology 77(2):198-209. https://doi.org/10.1111/his.14134
20. Santoriello D et al (2020) Postmortem kidney pathology findings in patients with COVID-19.J Am Soc Nephrol 31(9):2158-2167

21. Golmai P et al (2020) Histopathologic and ultrastructural findings in postmortem kidney biopsy material in 12 patients with AKI and COVID19. J Am Soc Nephrol 31(9):1944-1947

22. Sharma P et al (2020) COVID-19-associated kidney injury: a case series of kidney biopsy findings. J Am Soc Nephrol 31(9):1948-1958

23. Werion A et al (2020) SARS-coV-2 causes a specific dysfunction of the kidney proximal tubule. Kidney Int 98(5):1296-1307

24. Ronco C, Reis T (2020) Kidney involvement in COVID-19 and rationale for extracorporeal therapies. Nat Rev Nephrol 16(6):308-310. https:// doi.org/10.1038/s41581-020-0284-7

25. Risitano AM et al (2020) Complement as a target in COVID-19? Nat Rev Immunol 20(6):343-344

26. Leppkes $M$ et al (2020) Vascular occlusion by neutrophil extracellular traps in COVID-19. EBioMedicine 58:102925

27. Schulte-Schrepping J et al (2020) Severe COVID19 is marked by a dysregulated myeloid cell compartment. Cell 182(6):1419-1440.e23

28. Shetty AA et al (2020) COVID-19-associated glomerular disease. J Am Soc Nephrol 32(1):33-40. https://doi.org/10.1681/ASN.2020060804

29. Nadim MK et al (2020) COVID-19-associated acute kidney injury: consensus report of the 25th acute disease quality initiative (ADQI) workgroup. Nat Rev Nephrol 16(12):747-764

30. Nadim MKet al (2020) Publisher correction: COVID 19-associated acute kidney injury: consensus report of the 25(th) acute disease quality initiative (ADQI) workgroup. Nat Rev Nephrol 16(12):765

31. Best Rocha A et al (2020) Detection of SARSCoV-2 in formalin-fixed paraffin-embedded tissue sections using commercially available reagents. Lab Invest 100:1485-1489

32. Braun $F$ et al (2020) SARS-CoV-2 renal tropism associates with acute kidney injury. Lancet 396(10251):597-598

33. Farkash EA, Wilson AM, Jentzen JM (2020) Ultrastructural evidence for direct renal infection with SARS-coV-2. J Am Soc Nephrol 31(8):1683-1687

34. Roufosse C et al (2020) Electron microscopic investigations in COVID-19: not all crowns are coronas. Kidney Int 98(2):505-506. https://doi. org/10.1016/j.kint.2020.05.012

35. Hopfer $\mathrm{H}$ et al (2020) Hunting coronavirus by transmission electron microscopy - a guide to SARS-CoV-2-associated ultrastructural pathology in COVID-19 tissues. Histopathology. https://doi. org/10.1111/his.14264

36. Amann K, Boor P, Wiech T (2020) COVID-19 und Nierenbefall. Nephrologe. https://doi.org/10. 1007/s11560-020-00469-9
Auch online Zugang zu allen Beiträgen Ihres Zeitschriftenabonnements

Wussten Sie, dass Sie als Abonnent*in dieser Zeitschrift automatisch Online-Zugriffsrechte auf das gesamte Beitragsarchiv und die CMEKurse haben?

Der Zugang zu Ihrer Online-Bibliothek und den CME-Kursen ist im Abonnement Ihrer Zeitschrift inbegriffen. Für den Zugang müssen Sie sich lediglich einmal über www.springermedizin.de/register registrieren.

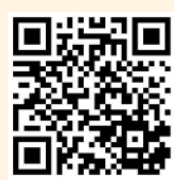

Über diesen QR-Code schnell und einfach registrieren

Bitte nutzen Sie für die Registrierung Vorund Nachname und Lieferadresse wie beim Abonnement der Zeitschrift (s. Adressaufkleber auf Ihrem Heft). So kann im System die Zugehörigkeit zu Ihrer Zeitschrift sichergestellt werden.

Aufgrund des Heilmittelwerbegesetzes dürfen die Inhalte der Website nur medizinischen Fachkreisen zur Verfügung gestellt werden. Bei der Anmeldung bitten wir Sie deshalb, einen Berufsnachweis vorzulegen. Bei einer Mitgliedschaft in der deutschen Ärztekammer reicht die Angabe der einheitliche Fortbildungsnummer (EFN). Alternativ schicken Sie eine Bestätigung des Arbeitgebers, Studiennachweis mit Angabe des Studiengangs ganz unkompliziert an kundenservice@springermedizin.de.

Mit Benutzername und Passwort haben Sie außerdem Zugang zu den freien Inhalten auf den Seiten von: www.springermedizin.de www.aerztezeitung.de

Sollten Fragen oder Probleme auftauchen, wenden Sie sich einfach an Ihren Kundenservice:

kundenservice@springermedizin.de 